\title{
ARTERIAL LESIONS FOUND IN PERSONS DYING FROM ACUTE INFECTIONS, AND ATTEMPTS TO PRO- DUCE ARTERIAL LESIONS IN ANIMALS BY NON-INFECTIOUS TOXINS *
}

\author{
CHANNING FROTHINGHAM, JR., M.D. \\ BOSTON
}

In a former study ${ }^{1}$ it was shown that diffuse arterial lesions of a mild grade were frequently associated with certain acute infectious diseases in young people. These lesions were in no way characteristic of acute infections, as they were similar to the usual lesions found in the arteries in old age, whatever be their causes. These lesions consisted of fatty changes with or without cellular invasion in the intima and media of the vessels. In some cases the connective tissue was increased in the intima, media or both, and in some there was loss of muscle substance in the media. In the arteries in the spleen there was a peculiar hyaline-like degeneration involving one or all the coats. It is impossible to say, therefore, whether or not these lesions in these special cases are due to the toxins of the disease.

In one of the cases of general septicemia in this former study a different kind of arterial lesion was found in the kidney. There was necrosis of the vessel wall with fibrin formation and invasion into and around the vessel wall of polynuclear leukocytes and endothelial cells, as is shown in the accompanying photograph.

It is known that the tubercle bacilli and the spirochetes of syphilis invade arterial walls and cause lesions characteristic of the organism, which leave a permanent scar. The question therefore arose as to the possibility of this lesion being due to the presence of some special infectious organism, or to a certain kind of circulating toxin.

This study consisted of a search for lesions of this type. For this purpose autopsy material, preserved in the usual routine manner of the Boston City Hospital pathological laboratory, was used. The tissue after Zenker's fixation was stained with eosin and methylene-blue stains. Sections from the more important organs and aorta were examined. Occasionally one or more organs were missing in a given case. Usually only one or two slides of the same organ were examined. This was found later

* Manuscript submitted for publication June 14, 1912.

* From the laboratory of the Department of Theory and Practice of Physic, Harvard University.

1. Frothingham: The ARChives IxT. MED., 1911, viii, 153. 
to have been unfortunate as in some cases lesions would only appear in every third or fourth slide.

As this type of injury to the vessel wall was quite distinct from the early or late so-called arteriosclerotic changes, tissue from patients up to 40 years of age was studied. In all, sections from fifty-six patients were examined. Of these forty-eight died from acute infectious diseases. These forty-eight cases included bronchopneumonia, diphtheria, typhoid fever, pneumonia, tuberculosis, scarlatina, measles, general sepsis, and septic conditions of the ear, salpinx, peritoneum, meninges, joints, brain. liver and pleura.

No mention will be made of the lesions in the ressels characteristic of so-called arteriosclerosis and occurring in cases of acute infection as described above. Only those lesions are mentioned in which there was necrosis of the vessel wall with fibrin formation and cellular invasion. In some of these ressels thrombi were formed which extended berond the site of the necrosis. In none of the eight cases which died from chronic non-infectious disorders were lesions of this type found.

of the forty-eight cases with acute infections eight showed arterial lesions of this type. These cases with the location of the lesions were as follows:

Artery in the kidney from the case numbered Path. Records 1910-15. For this photograph $I$ am indebted to Dr. F. B. Mallory.

Pathological record B. C. H. 1911-47, aged 6 years. Purulent meningitis. Autopsy cultures, streptococcus. Arteries in meninges involved. Arteries in other organs not affected.

Path. record B. C. H. 1911-38, aged 32 years, tuberculosis of lung and meninges. Autopsy cultures, tubercle bacilli, and mixed growth. Only meningeal vessels show this lesion.

Path. record B. C. H. 1911-42, aged 34 years. Acute endocarditis, pneumonia, pulmonary infarcts. No culture taken. This type of arterial lesion occurred only in the lung.

Path. record B. C. H. 1911-4, aged 3 years. Empyema and lung abscess with miliary tuberculosis following scarlet fever. Autopsy cultures showed mixed infection. Acute arterial lesions of this type found in liver and Iungs.

Path. record 1910-179, aged 3 years. Empyema, pericarditis, and meningitis following diphtheria. Autopsy cultures negative. This type of lesion only found in the vessels of the lung and meninges.

Path. record 1911-96, aged 3 vears. Diphtheria and bronchopneumonia. Autopsy cultures, diphtheria bacilli and others. Vessels in the pharyngeal wall showed these lesions.

Path. record 1910-15, aged 8 years. General sepsis with pus in joints, etc., following scarlet fever. No cultures taken. Marked arterial lesions of this type were found in the kidney, heart, liver, and muscle abscess.

Path. record, 1897.171 , aged 3 years. Diphtheria and interstitial nephritis. Autopsy cultures streptococeus and staphylococcus. Only tissue of the kidney was available. In the kidney were marked arterial lesions of this acute type.

From a study of these cases it is evident that marked arterial lesions of this type, although not common, do occur with moderate frequencr, and are pretty well distributed throughout the different organs. Ther 
seem to occur chiefly in infections caused by or complicated with the pusforming cocci. In no case could the possibjlity of the bacteria being present in the lesion be excluded, although they were not demonstrated. 'The case of periarteritis nodosa reported by Longcope shows a similar lesion and is considered to be due to some infectious process. Recently Mallory ${ }^{3}$ has found similar lesions in the arteries of the heart during acute articular rheumatism. The end-result of such a lesion on healing must show a permanent scar in the arterial wall. It seems reasonable

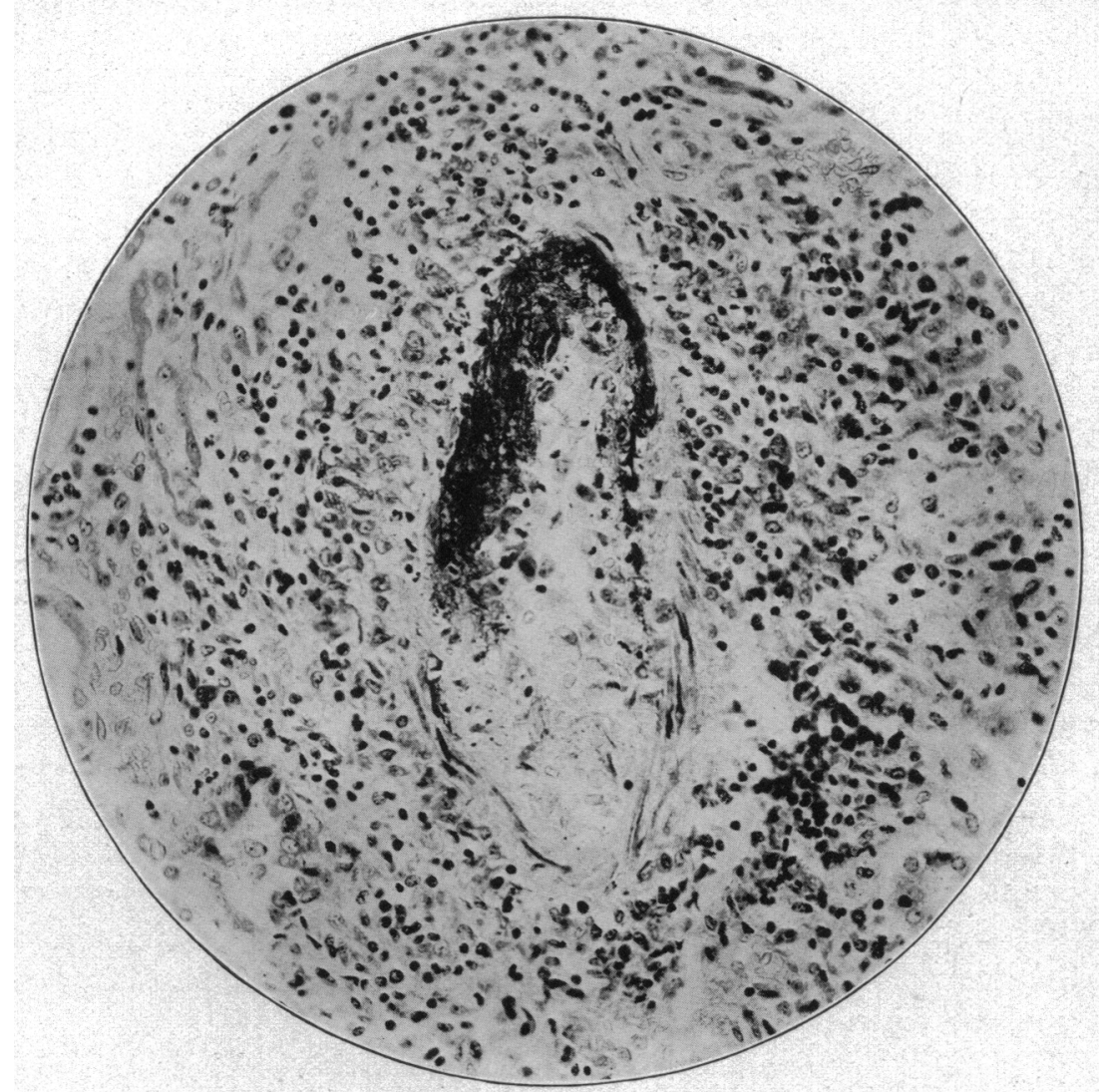

Marked arterial lesion found in an artery in the kidney following scarlet fever.

to suppose that less serere infections of the same type which terminate in recovery may cause similar arterial lesions with an arterial scar carried into later life. The question immediately arises, Do repeated lesions of this sort lead to the so-called arteriosclerosis of old age? If such a lesion heals with a partial occlusion of the lumen, it is reasonable to

2. Longcope: Bull. Ayer Clin. Lab., Fenn. Hosp., December, 1908.

3. Mallory, F. B.: Unpublished article. 
suppose that the vessel wall distal to this point may suffer from diminished blood-supply. It is also possible that the arterial wall adjacent to the scar may suffer from lack of nutrition due to the surrounding scar tissue.

With the hope that elinical histories might throw some light on the cause of arteriosclerosis the record of two patients with marked sclerosis of the arteries aged 22 and 16 years, respectively, were carefully studied. There was nothing in the history of either case which could be pointed to as a possible canse of the sclerosis. It is only fair to state that the histories were not carefully taken with this point in view.

In studying these scetions it was noted that in cases of chronic disorder such as nephritis, diabetes, ete, the arterial lesions characteristic of arteriosclerosis were usually quite marked. An attempt was made, therefore, to produce in rabbits arterial lesions of the degenerative type by metabolic or chemical poisons.

Although the experiments were uniformly unsuccessful in producing lesions, they will be briefly described. In this work tissues, preserved in formaldehyd solution, were stained with Scharlach $R$ for fat in addition to the routine eosin and methylene-blue stain on Zenker fixed material used in this laboratory.

Double nephrectomy under ether anesthesia was performed on five rabbits which lived from twenty-four to forty-four hours after operation. In this time fatty changes had occurred in the heart muscle as described by Lewis, ${ }^{4}$ but no evidence was found of lesions in the arteries.

Bovine" bile was injected intracenously, intraperitoneally and subeutaneously in varying amounts into rabbits for different lengths of time. Although the results varied with the different biles used il seemed as though fatty changes were produced in the heart and liver in some cases. No arterial lesions were formed, however, in any of the cases. Tissue from a cat which had been kept in a state of glycosuria for over a year by Dr. Allen was examined for fatty changes in the ressels. No pieces of the aorta were obtainable. but the arteries in the different organs showed no degenerative lesions.

The kidneys were examined in four rabbits which had been injected by Dr. Christian ${ }^{6}$ with uranium nitrate and either caffein or adrenalin and spartein in work on experimental nephritis. In these kidneys marked lesions were found in the parenchymal cells and also in the glomeruli. Hemorrhages were present in the glomeruli. In three of these cases no arterial lesions were found. In one a few fat granules were present in a few of the small arteries.

4. Lewis: Jour. Med. Research, 1907. xvii, 291.

5. Frothingham and Minot: Jour. Med. Research, 1912, xxvii.

6. Christian, Smith and Walker: The Archives Int. Med., 1911, viii, 468. 
In three rabbits which had been injected with adrenalin and spartein by Dr. Christian there were marked degenerative lesions in the cardiac muscle and proliferation of the cardiac connective tissue. In these cases the arteries showed no lesion with the possible exception of a slight amount of fat in a few arteries in one case. It is interesting to note that toxins, which will produce an hypertrophy of connective tissue in the stroma of an organ, apparently have no effect on the connective tissue of the vessel walls.

\section{CONCLUSION}

Although this study has thrown no light on the relation between noninfectious toxins and arterial disease it has shown that during acute infectious diseases severe localized arterial lesions may occur.

51 Hereford Street. 